\title{
Target-Costing - ein neues Planungsinstrument oder Rechnungssystem zur Planung
}

\author{
Prof. Dr. rer. oec. habil. Lothar Brunsch
}

\section{Einführung}

Seit einiger Zeit gibt es eine Diskussion in der Literatur, die sich unter dem Stichwort „Neuorientierung“ des internen Rechnungswesens zusammenfassen läßt. ${ }^{1}$ Hierzu gehören die Diskussion der spezifischen Rolle des Zielkostenmanagements bzw. Target-Costing im Rahmen der Kostenrechnungssysteme, speziell für die Kostenplanung bei Neuprodukten. ${ }^{2}$

Dieser Beitrag stellt sich das Ziel, aufbauend auf der aktuellen Diskussion, besonders die Einbindung des Zielkostenmanagements in andere Instrumente und Methoden der BWL zu beleuchten und überblicksartig auf die Gründe zur Herausbildung des Zielkostenmanagements einzugehen. In weiteren Beiträgen wird an dieser Stelle auf die Methodologie des Target-CostingProzesses und den Einfluß des Zulieferpotentials unter Einbeziehung der in einem Unternehmen des Schienenfahrzeugbaus gemachten Erfahrungen verwiesen. In diesen Beiträgen wird der englische Begriff „TargetCosting" mit Zielkostenrechnung bzw. präziser und umfassender mit Zielkostenmanagement uibersetzt bzw. gleichgestellt. Weitgefaßt wird unter Zielkostenmanagement ein „markt- bzw. kundenorientiertes Konzept der Kostenplanung, Kostensteuerung und Kostenkontrolle"3 verstanden. In Japan wird meistens für TargetCosting der Begriff „Genka-Kikaku“4 genutzt.

\section{Einordnung des Zielkostenmanagement}

Die Märkte, in denen sich Unternehmen bewegen, sind für sie der Fokus, in dem unternehmensinterne und unternehmensexterne Faktoren zusammentreffen und in welchem sie sich zu behaupten haben. Die rasche Entwicklung in vielen Entscheidungsbereichen der Technologie und Technik und der unternehmerischen Nutzung dieser Möglichkeiten führen unter anderem zu einer Globalisierung, Dynamik, Diskontinuität und Differenzierung der Märkte in einem bisher nicht gekannten Ausmaß. Neben dem Markt machen die Gesellschaft und der Staat diese Umweltfaktoren transparent. ${ }^{5}$

Diese Entwicklung findet ihren Ausdruck in wettbewerbsintensiveren Märkten. Für die Unternehmen steht die Aufgabe, ausgehend von ihren Zielen analog, dynamisch, global und differenziert auf diese Herausforderung zu reagieren; und das so, daß sie nicht nur auf den Märkten reagieren, sondern auf diesen agieren und sie gezielt beeinflussen. Auf diese Herausforderungen der Märkte haben die Unternehmen bereits Ende der 70er Jahre begonnen, unter anderem mit sehr verschiedenen innerbetrieblichen technologischen Konsequenzen zu reagieren (vgl. dazu Abb. 1).

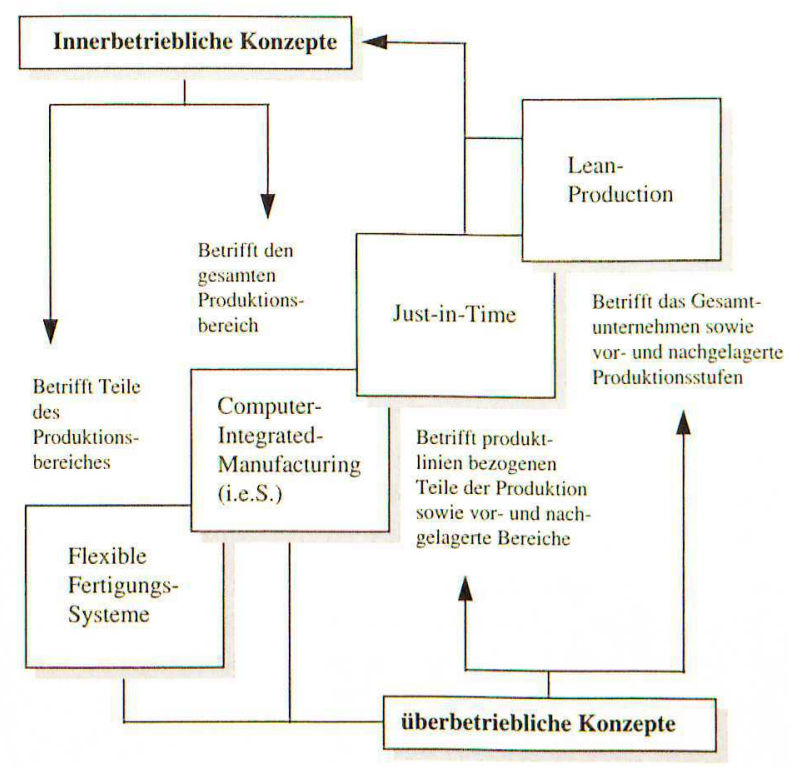

Abb. 1: Hierarchiesierung relevanter technologischer Neuentwicklungen, Larson, S. 457

Dabei stellt das Lean Production-Konzept einen Kerngedanken dar, welcher auf die Optimierung der gesamten uiber- und innerbetrieblichen logistischen Ketten des Organisations- bzw. Operationskonzeptes abzielt. ${ }^{6}$ In der Lean Production ist das Target-Costing ein Instrument zur Bewertung der in den einzelnen Produktionsstufen erzeugten Produkte bzw. Leistungen aus der Sicht des Marktes. Dies erfolgt, indem eine verstärkte Einbeziehung der Kostenkalkulation in Konstruktionsund Technologieerarbeitung im Rahmen der CAD/CAMund CIM-Anwendung durchgesetzt wird. Bei dieser methodischen Einordnung präsentiert sich das TargetCosting als strategisches Instrument, als ein Spezialfall der konstruktionsbegleitenden Kostenrechnung. Das Target-Costing erfaßt hier als Zielkostenmanagement sowohl den Marktzyklus als auch den gesamten Produktionslebenszyklus. Weitere Reaktionen der Unternehmen auf die oben beschriebene Entwicklung sind Entstehen und der Ausbau von Logistik, TQM, Marketing, das Controlling usw. In der Kostenrechnung gab es in den westlichen Ländern erst Ende der 80er Jahre eine Reaktion auf diese wesentlichen Marktveränderungen in Form der Entstehung des Activity Based Costing, der Prozeßkostenrechnung und des Target-Costing.?

Das Target-Costing wird weiterhin in Forschung, Lehre und Praxis häufig auf dem Gebiet der Unternehmenspla- 
nung im Rahmen der strategischen Führung behandelt. Ein möglicher Ansatz fuir die Einordnung des TargetCosting in die Unternehmensplanung ist der Führungsprozeß auf der Grundlage der Entscheidungs- und Verhaltenstheorie als Willensbildungs- und Willensduchsetzungsprozeß, aus dem sich die Führungsaufgaben Planung, Steuerung und Kontrolle ergeben. Im Rahmen der Unternehmensplanung ist das Target-Costing Bestandteil der strategischen Planung. Die strategische Planung umfaßt als Kern die Produktprogrammplanung und die zu ihrer Realisierung notwendige Unternehmenspotentialplanung in Form der Sach- und Humanpotentiale/Potentialänderungen im Rahmen der Investitions- und Desinvestitionsplanung. ${ }^{8}$ In der strategischen Planung ist das Target-Costing ein Rechnungssystem, das innerhalb der strategischen Produkt- und Potentialplanung den Charakter eines Beurteilungsinstrumentes einnimmt. Es dient hierbei letztendlich dazu, aus kostenrechnerischer Sicht Aussagen zu Definitionen der Produktprogramme bzw. der Produktprogrammalternativen zu liefern. (vgl. Abb. 2)

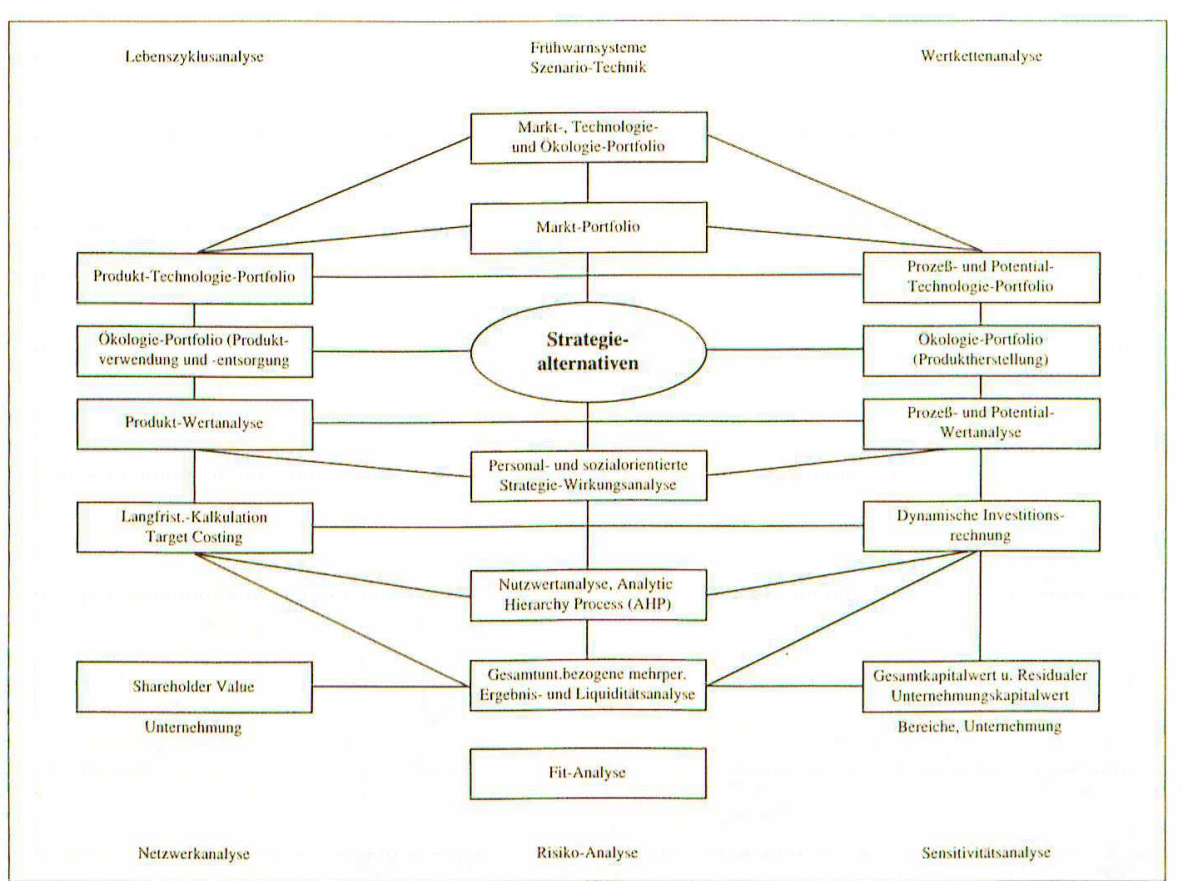

Abb. 2: Beurteilungsinstrumente für Strategiealternativen, Hahn, S. 296

Hierbei wird das Target-Costing als ein langfristiges Kalkulationsinstrument aufgefaßt, das neben den Attraktivitäts- und Wettbewerbsanalysen (Portfolio-Analysen) und der Nutzwertanalyse der Bewertung der Produktprogrammplanung dient. ${ }^{9}$

Gleichzeitig wird durch den Einsatz des Target-Costing eine aktive Planungs-, Steuerungs- und Kontrollfunktion hervorgerufen, die zur Bestimmung und Beeinflussung wettbewerbs- und marktorientierter Kosten und Preise im Rahmen gegebener Kapazitäten fuihrt. Durch diese Planungs-, Steuerungs- und Kontrollfunktion entsteht aus der Zielkostenrechnung verstärkt das Zielkostenmanagement. Mit dem Target-Costing ist eine Möglichkeit gegeben, für ein Produkt bzw. fuir ein Produktprogramm die geldlich ergebnisorientierten Auswirkungen der strategischen Alternativen sichtbar zu machen. Auf Unternehmensebene erfolgt die monetäre Darstellung durch die dynamischen Investitionsrechnungssysteme. Beide Rechnungssysteme stehen in einem engen Wechselverhältnis und bedingen einander. Werden Kosten primär durch Veränderung bzw. Umschichtung von Kapazitäten beeinflußt, z. B. durch Auslagerung von Potentialen, sind das Aktivitäten der Investitionssphäre, wobei, wie bereits dargestellt, Investitionsaktivitäten im Zusammenhang mit dem TargetCosting stehen. Zur Erhöhung bzw. Sicherung des Erfolgspotentials als dritte überlebensrelevante Steuerungsgröße (neben Liquidität und Rentabilität) wird das Target-Costing aus der Sicht der Kostenführerschaft betrachtet. Dabei hat die strategische Kostenanalyse mittels des Target-Costing die Aufgabe, eine Kostenfuihrerschaftsstrategie zu entwerfen und effizient voranzutreiben. ${ }^{10}$ Auch hier iibernimmt das Target-Costing die Funktion des Bewertungsinstrumentes.

Das Target-Costing eignet sich somit besonders für die Produktplanung der montierenden Produktion mit sich permanent verkürzenden Produktionszyklen, wie sie z. B. für den Fahrzeugbau und Schienenfahrzeugbau typisch sind, und wird auf beiden Gebieten seit mehreren Jahren angewandt. Der Vorreiter dazu war die Fahrzeugindustrie. ${ }^{11}$ Ein weiterer Ansatzpunkt fuir die Einordnung des Target-Costing in die Prozesse der Unternehmensfuihrung entsteht daraus, daß das Target-Costing auf die permanente Versorgung mit entscheidungsrelevanten und empfängerorientierten Informationen angewiesen ist, die es braucht, um die marktgemäße Entwicklung und Herstellung des Fertigungsgutes zu gewährleisten. Diese Information erhält das Target-Costing nur aus einem adäquat aufgebauten Controllingsystem. Adäquat heißt, daß dieses Controlling verstärkt verhaltensorientiert arbeitet und zum Abbau bzw. zur Verminderung der Unternehmenskomplexität beiträgt, in dem die Strukturen uiberschaubar, durchsichtig und auf rasches Reagieren hin gestaltet werden.

In Wechselbeziehung zum Target-Costing sollte das Controlling folgende Aufgaben übernehmen:

- als Initiator in den Projekten das Target-Costing durch seine Anwesenheit im Projektteam in Bewegung halten,

- fruihzeitig aktuelle entscheidungsrelevante Kosteninformationen zur Verfügung stellen.

Im Controlling sind die Daten - wie im Controlling allgemein üblich - zu analysieren, zu bewerten und Lösungswege zur E $\boldsymbol{r}^{3}$-ichung der Zielstellung vorzuschlagen. ${ }^{12}$ Ziel sollte es sein, auf ein Self-Controlling und auf 
ein marktnahes Denken der Mitarbeiter hinzuwirken. Diese kurze Auswahl von Einsatz- und Ansatzmöglichkeiten des Target-Costing in der Betriebswirtschaftslehre bzw. in der Unternehmenspraxis soll genügen, um auf die bereits erzielte breite Wirkung des Target-Costing hinzuweisen und um zu verdeutlichen, daß viele betriebswirtschaftliche traditionelle Methoden, Instrumente usw. in diesem Kontext neu zu durchdenken sind.

Aus den Ausfuihrungen wird deutlich, daß das TargetCosting vor allem auf folgenden Gebieten seine Wirkungsmöglichkeiten findet:

1. in der strategischen Planung, dem strategischen Controlling und der konstruktionsbegleitenden Kalkulation als Bewertungsinstrument zur Produktentwicklung und Produktplanung mit verschiedener Zielsetzung und zur fruihzeitigen Optimierung der Produktionsstrukturen;

2. in der Kostenrechnung als Zielkostenmanagement, wo mit ihm Maßnahmen zur Beeinflussung der Kosten im Sinne der Planung, Steuerung und Kontrolle durchgeführt werden. Dabei drückt das Zielkostenmanagement präziser aus, daß hierbei die Orientierung an den vom Markt vorgegebenen Anforderungen erfolgt. Besonders geht es um die produktbezogene Kostenbeeinflussung, ,die bereits in der Phase der Entwicklung und Konstruktion von Produkten ansetzt". 13 (vgl. Abb. 3)

An dieser Stelle ist darauf hinzuweisen, daß die Verwirklichung eines definierten Kostenniveaus nicht das oberste Ziel marktwirtschaftlich geführter Unternehmen ist. Das oberste Ziel der Kostenpolitik ist und bleibt die Erreichung des vorgegebenen übergeordneten Gewinnziels. Zur Verwirklichung dieser Zielstellung sind auch verstärkt Markt-Preis-Überlegungen mit einzubeziehen. Das Bestimmen von Marktpreisen ist besonders bei neu einzufuihrenden Produkten eine strategische Entscheidung, denn das hat starken Einfluß auf die Verwirklichung der verfolgten Marktziele. In diesem Kontext ist das Zielkostenmanagement eingebettet. Die Zielkosten werden aus dem langfristigen Gewinnplan des Unternehmens abgeleitet ${ }^{14}$ und dem Konstrukteur wie dem

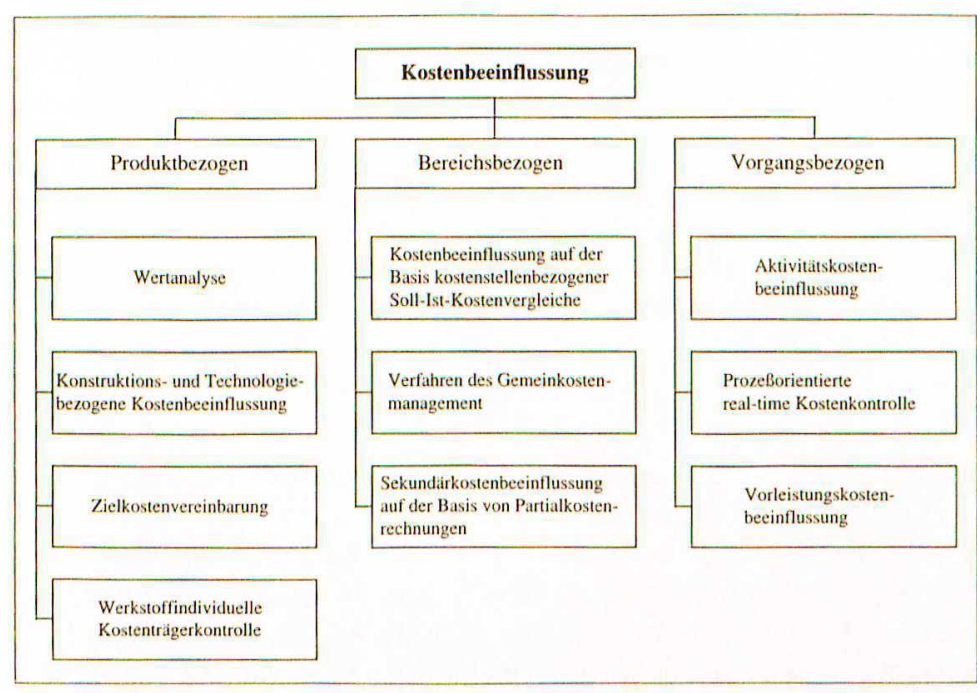

Abb. 3: Die Formen der Kostenbeeinflussung, vgl. Franz, 1994
Verantwortlichen für die Entwicklung der Produkte und Leistungen als Leitlinien für ihre Tätigkeit vorgegeben. „Zweck des Zielkostenmanagement ist die Einordnung produktbezogener Kostenziele in die langfristigen Gewinnziele der Unternehmung in einer Weise, daß die für die Kosten in erster Linie verantwortlichen Personen operable Zielvorstellungen fuir ihren Arbeitsbereich erhalten."15

Davon ausgehend können unter Target-Costs „die durchschnittlich geplanten Selbstkosten für eine Produkteinheit oder eine Produktgruppe verstanden werden, die vom Management als Zwischenziel auf dem Weg zur Erreichung der Allowable-Costs angesetzt werden. Die Allowable-Costs erlauben den erfolgreichen Markteintritt oder Verbleib und ergeben sich durch Reduktion des Zielpreises um einen geplanten Gewinnanteil." 16

Die Target-Costings haben damit „im gesamten Leistungserstellungsprozeß sowohl eine implizit sachliche als auch eine wertmäßige Orientierungsfunktion für die Mitarbeiter"17, womit der Stellenwert der verhaltensbezogenen Komponente des Konzepts hervorgehoben wird.

Im folgenden ist darauf einzugehen, warum die traditionelle Kostenrechnung diese Anforderungen nicht erfüllen kann bzw. warum sie für diese Aufgaben nicht konzipiert wurde.

\section{Grenzen der traditionellen Kostenrechnung bei der Kostenplanung}

Im Gegensatz zum Target-Costing wurde die traditionelle Kostenrechnung, die unter den Bedingungen der taylorischen Produktionsstrukturen und der Massenfertigung entstand, als laufende periodische und kalkulatorische Rechnung dargestellt. Das findet seine Widerspiegelung in der klassischen und in der neueren Vollkostenrechnung und in der Teilkostenrechnung, die im wesentlichen auf Vergangenheitswerten beruhen, aber auch begrenzt in der Grenzplankostenrechnung. Die traditionelle Kostenrechnung stellt den betrieblichen Werteverzehr sowie die Entstehung dieser Werte dar. ${ }^{18}$ Durch ihre periodische Abgrenzung unterscheidet sich die Kostenrechnung von der Investitionsrechnung. Als einziges Kostenrechnungskonzept läßt sich von diesem Periodisierungspostulat das „Riebel'sche“ Einzelkostenrechnungssystem nicht leiten. Diesem Weg folgt das Target-Costing-Rechnungskonzept und läßt sich deshalb auch dem Planungssystem zurechnen. ${ }^{19}$

Bei der Kalkulation von Neuprodukten wird bei der traditionellen Kostenrechnung von den voraussichtlichen und tatsächlichen Kosten seitens der Absatzleistung ausgegangen, wobei die Herstell- und Selbstkosten die Preisuntergrenze darstellen. ${ }^{20}$ Hierbei handelt es sich um die klassische Vollkostenkalkulation, deren Mängel hinlänglich bekannt sind. Aus der Sicht der Kalkulation neuer Produkte sei aber darauf hingewiesen, daß 
sich die Kritik an der traditionellen Kostenplanung in Form der Plankostenrechnung vor allem auf ihren späten Einsatzpunkt, d. h. nach dem Abschluß der Konstruktion bezieht. Sollte sich bei der Kalkulation herausstellen, daß das Produkt überteuert ist, besteht lediglich die Möglichkeit, die Konstruktionsabteilung mit der Überarbeitung der Konstruktionszeichnung zu beauftragen, wobei Veränderungen im eigentlichen Sinne nur noch bedingt möglich sind. Besonders bei Projekten und Produkten, die mit Baugruppen und Einzelgruppen gebaut werden, sind Einflußmöglichkeiten zu diesem Zeitpunkt mit dem vertretbaren Aufwand nur noch begrenzt gegeben.

„Die Vorgehensweise bedingt den Durchlauf eines langen Regelkreises, der die ökonomische Beurteilung des neuen Produktes an das Ende des gesamten Produktentwicklungsprozesses stellt. Gerade angesichts des enormen Wettbewerbsdrucks, der die Unternehmen zur Beruicksichtigung von Qualitäts- und Preiserwartung des Kunden unter gleichzeitiger Beachtung des Zeithorizontes bis zur Markteinfuihrung zwingt, sind die traditionellen Methoden als nicht zufriedenstellende Lösung anzusehen."21

Das traditionelle Kostenmanagement orientiert sich am klassisch führungsorientierten Rechnungswesen und versucht nur im Rahmen der vorgegebenen bzw. vorhandenen Strukturen die Kosten zu optimieren. Die Mängel des traditionellen Kostenmanagements, bezogen auf die heutigen Anforderungen, lassen sich in folgender Art darstellen:

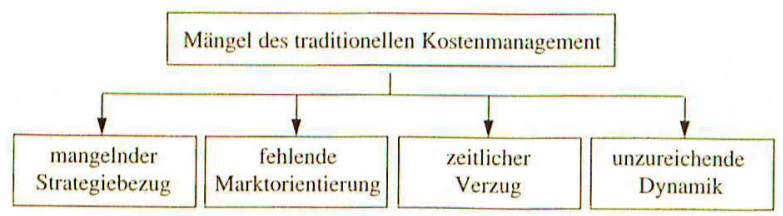

Abb. 4: Mängel des traditionellen Kostenmanagements, vgl. Seidenschwarz, 1993

\section{Mangelnder Strategiebezug}

Die traditionelle Kostenrechnung orientiert sich nicht ausreichend auf die Unterstuitzung der Produktpolitik. Dazu müßte sie Informationen über die langfristige Gesamtrentabilität der Produkte liefern und die Kostenentwicklung über den gesamten Produktlebenszyklus darstellen, einschließlich der Nachlaufkosten. Dazu wäre eine Orientierung am Markt erforderlich.

\section{Fehlende Marktorientierung}

Durch die bereits oben erwähnte Neuentwicklung von Produktions- und Informationstechnologien kommt es zu einem Ansteigen der Kompliziertheit betrieblicher Strukturen und Abläufe. Weiterhin fuihrt diese Entwicklung zu einem sinkenden Anteil der direkten Kosten an den Gesamtkosten bei gleichzeitig rasch zunehmenden indirekten Kosten.22 Das führt dazu, daß ein auf den Einzelkosten der Fertigung basierendes Kostenrechnungssystem seine Aussagekraft für ein marktorientiertes Kostenmanagement verliert. ${ }^{23}$ Hiromoto spricht hier von einem technologiegesteuerten Gegensatz zu einem anzustrebenden marktgesteuerten Kostenmanagement. ${ }^{24}$
Benötigt wird ein Kostenmanagement, das eine Verbindung zwischen dem externen und internen Unternehmensfeld gewährleistet, um aufzuzeigen, welche unternehmensinternen Konsequenzen sich aus den Marktanforderungen ergeben. Es gilt, die Marktanforderungen intensiv mit den technologischen Möglichkeiten des Unternehmens abzugleichen, um zu verhindern, daß im Unternehmen vorherrschende und installierte Technologien produktbestimmend sind und es damit zum "Over-Engineering" kommt.

Die Erfüllung der vom Kunden im Vertrag geforderten Funktionen sind der Maßstab, nicht die Funktionsübererfuillung. Denn diese führt zu notwendigen Mehrkosten und werden vom Kunden in der Regel nicht beglichen. Ein modernes Kostenmanagement ist deshalb darauf ausgerichtet, daß die Preise das Niveau der am Markt durchsetzbaren Kosten widerspiegeln und nicht, daß die Kosten das Preisniveau bestimmen. ${ }^{25}$

\section{Zeitlicher Verzug}

Die traditionellen Kostenrechnungskonzepte liefern eine mangelnde Unterstiitzung der Konstruktion. ${ }^{26}$ Diese Unterstiitzung kann die traditionelle Kostenrechnung deshalb nicht geben, weil der Produktentstehungsprozeß von technologieorientierten Produkten sequentiell abläuft. (vgl. Abb. 5)
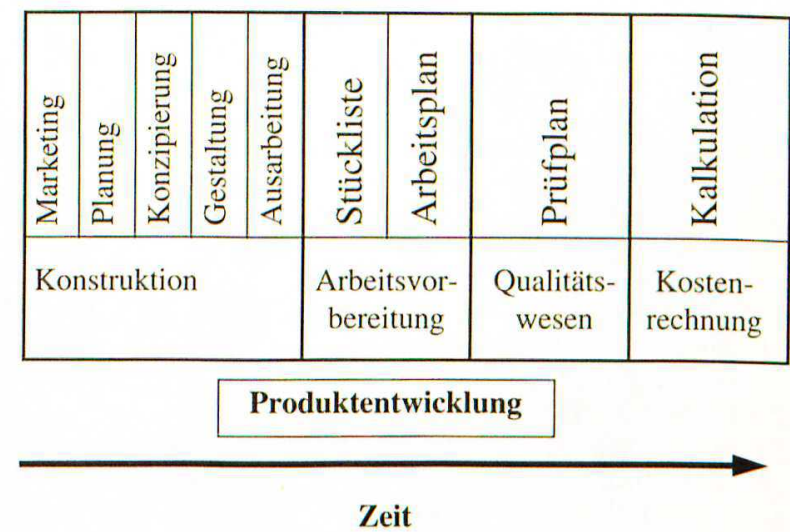

Abb. 5: Sequentielles Entwicklungsmanagement, Scheer, 1989, S. 7

Dieses Vorgehen verhindert eine frühzeitige Steuerung der Produktkosten. Gefordert werden Kalkulationsverfahren, die die Fähigkeit zur interdisziplinären Leitung in den verschiedenen Projektschritten einer Neuentwicklung besitzen und sich durch ihre fruihzeitige Einsatzmöglichkeit auszeichnen. ${ }^{27}$

\section{Unzureichende Dynamik}

Die Kostenrechnung muß frühzeitig Kostengesichtspunkte in den Konstruktionsprozeß einleiten und so an der Kostengestaltung maßgeblich mitwirken. Gefordert wird eine Dynamisierung der Kostenentwicklung unter Beachtung sämtlicher Einflußpotentiale während der Konstruktion.

Aus dieser Kritik ergeben sich folgende Anforderungen an ein modernes Kostenmanagement:

- eine Kostenplanung unabhängig von den Produktionstechnologien des Unternehmens, d. h., die Produkt- als auch die Produktionstechnologien sind auf 
die Erfüllung der Kundenwünsche abzustimmen;

- die Kostenplanung muß die strategischen Gesichtspunkte beachten und eine ganzheitliche Sichtweise gewährleisten;

- bei der Entwicklung von neuen Produkten ist frühzeitig die Kostenrechnung einzusetzen, damit in der Konstruktionsphase die Kosteninformationen fuir Produktvarianten einfließen können;

- die Kostenrechnung muß zunehmend motivationalen Aspekten Rechnung tragen;

- ein Cost-Management muß, wie bereits erwähnt, den Anspruch der Dynamik erfüllen. ${ }^{28}$

\section{Anmerkungen}

1 Stellvertretend seien für diese Diskussion genannt: Männel, 1993; Pfaff, 1994; Weber 1995

2 vgl. Horwáth und Seidenschwarz, 1991

3 Schneider, 1992, S. 293

4 vgl. Hiromoto, 1989, S. $320 \mathrm{ff}$

5 vgl. Weber, 1995, S. 567

6 vgl. Larson, 1993, S. 458

7 vgl. Weber, 1995, S. 566; Becker, 1993, S. 10-17; Franz, 1994

8 vgl. Hahn, 1995, S. 267

9 vgl. Hahn, S. $289 f$

10 vgl. Steinmann, S. 1460; Guilweiler, 1977, S. 67-75

11 vgl. Ziegler, 1994, S. 175-188

12 vgl. Horváth etal, 1993; Döpper, 1992; Buggert, Wielpuitz, 1995

13 vgl. Franz, 1992, S. $1493 f$

14 vgl. Makido, 1989

15 Franz, 1992, S. 1500

16 Buggert, Wielpuitz, S. 41

17 Pfeifer, Weiß, 1992, S. 215

18 Schweitzer, 1983, S. 682 „Unter Kostenrechnung versteht man ein System von Rechnungsverfahren, mit deren Hilfe bestimmte Kosten unter spezifischer Zwecksetzung bestimmten Bezugsgrössen zugerechnet werden."

19 vgl. Weber, 1995, S. 3

20 vgl. Männel, 1992, S. 340; Kilger, 1988, S. 605

21 Buggert, Wielpuitz S. 24

22 vgl. Buggert, Wielpititz, S. 28

23 vgl. Horváth, 1990, S. 177

24 vgl. Hiromoto, 1991, S. 35

25 vgl. Männel, 1993, S. 75

26 vgl. Männel, 1994, S. 106 ff

27 vgl. Becker, 1990, S. 353

28 vgl. Buggert, Wielpuitz, S. 37-39

\section{Literaturverzeichnis}

Beehen, J.: Entwurfs- und konstruktionsbegleitende Kalkulation, in: KVP, Nr. 6, 1990, S. 353-358

Betz, St.: Die Erfahrungskurve als Instrument der Zielkostenspaltung, in: BFuP, 6/95

Betz, St.: Die Erfahrungskurve als Instrument der Zielkostenspaltung, Herne/Berlin, in: BFuP, 47. Jg., 1995, S. 609-625

Buggert, W.; Wielpütz, A.: Target-Costing, 1. Auflage, München, Wien 1995

Buggert, W.; Wielpütz, A.: Target Costing - Gundlagen und Umsetzung des Zielkostenmanagement, in: Hanser Verlag, München Wien, 1995

Deutsche Norm: Berechnungsgrundlagen, Kalkulationsarten und -verfahren, in: DIN 32992, Teil 1, Dezember 1989

Döpper, K.: Targetorientiertes Controlling bei der Toshiba in Europa, in: Effektives schlankes Controlling, Hrsg.: Horváth, P., Stuttgart, 1992, S. 245-259

Franz, K.-P.: Moderne Methoden der Kostenbeeinflussung, in:
Handbuch Kostenrechnung, Hrsg.: Männel, W., Wiesbaden 1992, S. 1492-1505

Franz, K.-P.: Möglichkeiten eines systematischen Kostenmanagements, in: Scheer, A.W. (Hrsg.): Rechnungswesen und EDV, 15. Saarbruicker Arbeitstagung, Heidelberg, 1994, S. 59-71 Gülweiler, A.: Steuerung der Kostenhöhe und Kostenstruktur durch strategische Planung, in: Die Betriebswirtschaft, 37. Jg., 1977, S. 67-75

Hahn, D.: PuK-Controllingkonzepte, 5. Auflage, Wiesbaden, 1996

Hartmann, H.: Materialwirtschaft - Organisation, Planung, Durchfuihrung, Kontrolle, in: 6. uiberarb. u. erw. Auflage, Taylorix Fachverlag, Stuttgart, 1993

Heim, W.: Outsourcing - wettbewerbsfähiger durch optimale Nutzung der Potentiale von Zulieferern, in: Management Zeitschrift, 63, 1994, 7/8

Heinmann, H.; Guthunz, U.; Hasselberg, F.: Kostenfuihrerschaft und Kostenrechnung, in: Handbuch der Kostenrechnung, Hrsg.: Männel, W., Wiesbaden, 1992, S. 1459-1477

Hiromoto, T.: Wie das Management Accounting seine Bedeutung zurïckgewinnt, in: IFUA, Horváth \& Partner GmbH (Hrsg.), 1991, S. 25-46

Horváth, P.: Revolution im Rechnungswesen - Strategisches Kostenmanagement, in: Horváth, P. (Hrsg.), 1990, S. 175-193 Horváth, P.; Herter, R.N.: Benchmarketing, in: Controlling 4, 1992, S. 4-11

Horváth, P.; Kieninger, M.; Mayer, R.; Schimank, Ch.: Prozeßkostenrechnung - oder wie die Praxis die Theorie überholt, in: DBW, 53. Jg. , 1993, S. 609-628

Horváth, P.; Seidenschwarz, W.: Die Methodik des Zielkostenanagements, Controlling-Forschungsbericht Nr. 33 des Lehrstuhls Controlling am Betriebswirtschaftlichen Institut der Universität Stuttgart, 1992

Horváth, P.; Seidenschwarz, W.: Die Methodik des Zielkostenmanagement, in: Controlling Forschungsbericht Nr. 33 des Lehrstuhls Controlling der Universität Stuttgart, Stuttgart, 1991

Katja, M.: Cost Management System in Nissan and Application in Europe, Vortrag auf dem Kongreß „Target Costing“ 28.9.1992, Frankfurt

Kilger: Flexible Plankostenrechnung und Deckungsbeitragsrechnung, 9. Auflage, Wiesbaden, 1988

Köster, T.; Lohse, A.: Konzept eines MaterialwirtschaftsInformationssystems (MAWIS); Institut für Angewandte Produktionstechnologie, IAP GmbH, Braunschweig, November 1995

Krokowski, W.: Total Cost of Ownership (TCO) - ein unterstuitzendes Instrument zur Lieferantenauswahl im Bereich der Beschaffungslogistik, in: RKW-Handbuch Logistik, 18. Lfg., $1 / 1993$

Larson, P.: Zukunftsaufgaben des Rechnungswesens, in: Betrieb und Wirtschaft, Heft 14, 1993, S. 457-461

Männel, W.: Moderne Konzepte für Kostenrechnung, Controlling und Kostenmanagement, in: KVP, Nr. 2, 1993, S. 69-78

Männel, W.: Bedeutsame Ansätze, Konzepte und Instrumente als Kostenmanagement, in: KVP, Nr. 6, 1992, S. 340-343

Männel, W.: Fruihzeitige Kostenkalkulation und lebenszyklusbezogene Ergebnisrechnung, in: KVP, Nr. 2, 1994, S. 106-110 Neuber, A.: Entwicklung einer Konzeption zu Einfuihrung des Zielkostenmanagements für kundenspezifische Auftragsfertiger, in: Konstruktiver Entwurf, Institut für Werkzeugmaschinen und Fertigungs-technik, Technische Universität Braunschweig, September 1995

o. A.: AEG Handbuch Projektmanagement

Pfaff, D.: Zur Notwendigkeit einer eigenständigen Kostenrechnung, in: ZfbF, 46. Jg., 1994, S. 1065-1084

Sakurai, M.: Target Costing and How to Use It, in: Journal of Cost Management (3), 1989, S. 39-50 
Scheer, A.W: Information Management bei der Produktentwicklung, in: Information Management, Nr. 3, 1989, S. 6-11 Schweitzer, M.: Kostenrechnung, in: Management-Enzyklopädie, 2. Auflage, Bd. 5, Landsberg/Lech, 1983, S. 682-699 Seidenschwarz, W.: Target Costing - Schnittstellenbewältigung mit Zielkosten, in: Synergien durch Schnittstellen-Controlling, Hrsg.: Horváth, P., 1991, S. 198-207

Seidenschwarz, W.: Target Costing, München, 1993

Seidenschwarz, W.: Target Costing. Ein japanischer Ansatz für das Kostenmanagement, in: Controlling, 1991, S. 199

Seidenschwarz, W.: Target Costing - marktorientiertes Zielkostenmanagement, in: Verlag Franz Vahlen, München, 1993 Seidenschwarz, W.: Target Costing und Prozeßkostenrechnung, in: Prozeßkostenmanagement, München, 1991

Tanaka, M.: Cost Planing an Control Systems in the Design Phase of a New Product, in: Monden, Yasuhiro und Sakurai, Miehiaru (Hrsg.): Japanese Management Accounting - A Wold Class Approach to Profit Management, Cambridge/Mussachusetts, 1989, S. 49-71

Weber, J.: Kostenrechnungs-Dynamik - Einfluisse hoher unternehmensex- und -interner Veränderungen auf die Gestaltung, in: BFuP, 47. Jg., S. 65-581

Ziegler, H.: Neuorientierung des internen Rechnungswesens für das Unternehmenscontrolling im Hause Siemens, in: ZfbF, 46. Jg., 1994, S. 175-188

\section{Verfasser}

Prof. Dr. rer. oec. habil. Lothar Brunsch

Technische Fachhochschule Wildau

Fachbereich Betriebswirtschaft/Wirtschaftsinformatik Tel. (0 33 75) 508- 950 